\title{
FACTORS INFLUENCING MENTAL HEALTH IN ZAMBIAN ADOLESCENTS WITH HIV AND
} AIDS

\author{
S. Degun ${ }^{1}$, A. Menon ${ }^{2}$, A. McPherson ${ }^{3}$, M. Ngoma ${ }^{4}$, R. Nair ${ }^{5}$, J. Andren ${ }^{1}$, P. Garrud ${ }^{6}$, C. Glazebrook ${ }^{1}$ \\ ${ }^{1}$ Psychiatry, University of Nottingham, Nottingham, UK, ${ }^{2}$ Psychology, Unniversity of Zambia, Lusaka, \\ Zambia, ${ }^{3}$ Bloorview Research Institute, Holland Bloorview Kids Rehabilitation Hospital, Toronto, ON, \\ Canada, ${ }^{4}$ Child Health, University Teaching Hospital, Lusaka, Zambia, ${ }^{5}$ Institute of Work Health and \\ Organisations, University of Nottingham, Nottingham, ${ }^{6}$ School of Graduate Entry Medicine and Health, \\ University of Nottingham, Derby, $U K$
}

Background and aims: In developing countries information provision for young people about HIV and AIDs has tended to focus on prevention. Since knowledge underpins effective self-management of chronic conditions, poor knowledge about HIV could contribute to the psychological morbidity. This study aims to explore the relationship between knowledge of HIV and mental health of Zambian adolescents living with HIV and AIDS.

Methods: Participants were recruited to this cross-sectional survey if they were aged 11 to 15 years and attending clinics in the Lusaka region for management of HIV and AIDS. Participants completed questionnaires assessing HIV knowledge, HIV related stigma and mental health (Strengths and Difficulties Questionnaire - SDQ).

Results: Of 75 participants recruited to the study (51.9\% female), 28 (37.3\%) scored above the cut-off for total SDQ score suggesting possible or probable disorder: three times that found in a normative UK sample $(\mathrm{OR}=3.0, \mathrm{CI}=1.5$ to 5.8$)$. Levels of peer problems were high compared to $\mathrm{UK}$ norms $(\mathrm{OR}=9.1, \mathrm{CI}=4.2$ to $20)$ and a sample of Zambian school children aged 11 to $15(\mathrm{OR}=1.79, \mathrm{CI}=1.08$ to 2.9). Higher total SDQ scores were associated with younger age $(\mathrm{p}=0.03)$, lower levels of knowledge about HIV and AIDS ( $<<$ $0.001)$ and higher levels of HIV related stigma $(\mathrm{P}<0.001)$. Stepwise regression found that HIV knowledge independently explained $35 \%$ of variance in SDQ scores.

Conclusions: The results from this study highlight the mental health needs of young people with HIV in a developing country and suggest a need for appropriate patient education. 